Research Article

\title{
Reconstruction of Input Excitation Acting on Vibration Isolation System
}

\author{
Pan Zhou, ${ }^{1}$ Wen L. Li, ${ }^{2}$ Wanyou Li, ${ }^{1}$ and Zhijun Shuai ${ }^{1}$ \\ ${ }^{1}$ College of Power and Energy Engineering, Harbin Engineering University, Harbin 150001, China \\ ${ }^{2}$ Advanced Engineering and Technologies, Canton, MI 48187, USA \\ Correspondence should be addressed to Wanyou Li; hrbeu_ripet_lwy@163.com
}

Received 2 April 2016; Revised 31 July 2016; Accepted 10 August 2016

Academic Editor: Lei Zuo

Copyright (c) 2016 Pan Zhou et al. This is an open access article distributed under the Creative Commons Attribution License, which permits unrestricted use, distribution, and reproduction in any medium, provided the original work is properly cited.

\begin{abstract}
Vibration isolation systems are widely employed in automotive, marine, aerospace, and other engineering fields. Accurate input forces are of great significance for mechanical design, vibration prediction, and structure modification and optimization. Onestage vibration isolation system including engine, vibration isolators, and flexible supporting structure is modeled theoretically in this paper. Input excitation acting on the vibration isolation system is reconstructed using dynamic responses measured on engine and supporting structure under in-suit condition. The reconstructed forces reveal that dynamic responses on rigid body are likely to provide more accurate estimation results. Moreover, in order to improve the accuracy of excitation reconstructed by dynamic responses on flexible supporting structure, auto/cross-power spectral density function is utilized to reduce measurement noise.
\end{abstract}

\section{Introduction}

Vibration responses of mechanical equipment are frequently used for fault diagnosis in engineering applications [13]. Since installation impedances of equipment in different environments change significantly, the resultant vibration responses will be different accordingly. Therefore, it is necessary to adopt another physical quantity as diagnostic criterion. Input excitation relatively stays stable once the mechanical equipment is operating in steady conditions. The use of excitation force for fault diagnosis enables one to unify diagnostic criterion for the same equipment in different installation environments.

Taking diesel engine as an example, it is installed on the elastic hull structure on the ship. However, its delivery test is carried on when it is installed on stiff and rigid foundation. Dynamic responses of the engine installed on ship are totally different from those installed on land. It is very hard to measure directly excitation forces inside the engine. In such a situation, dynamic responses are applied to determine the internal excitation acting on diesel engine. In general, diesel engine is connected with hull structure through vibration isolators the use of which can obviously reduce vibration transmitted to hull structure [4]. This paper aims to discuss the reconstruction of excitation force acting on mechanical structure installed on flexible supporting through vibration isolators and to provide a theoretical basis for engineering applications.

Input reconstruction, providing significant basis for mechanical structure design, vibration prediction, structure modification, and fault diagnosis, has received extensive attention in recent years [5-7]. Input determination problem is actually an inverse problem solved based on the known system characteristics, that is, transfer functions and dynamic responses of mechanical system. As we all know, inverse problem is likely to be ill-posed and small measurement errors in the transfer functions and dynamic responses will result in largely amplified errors in the estimated excitation due to matrix inversion $[8,9]$. Various regularization technologies are proposed to decrease the influence of noise and improve the accuracy of least squares solution [10-12]. Singular Value Decomposition (SVD) is widely applied to calculate the pseudoinverse of transfer function matrix in inverse problem $[13,14]$. Truncated SVD is employed to discard small singular values of transfer function matrix to stabilize the solution $[11,15]$. Another popular approach is Tikhonov regularization method in which any small singular values will be effectively increased by the addition of the 
regularization parameter and the inversion of the matrix is thus stabilized $[16,17]$. These approaches are categorized as direct regularization methods by Hansen [18]. And the second category is known as iterative regularization method, including Conjugate Gradients [19-21] and LSQR $[18,22]$.

There is no doubt that the previous researches are of great value for inverse problem. Moreover, it is worth noting that the methods mentioned above are utilized on the basis of measured transfer functions between input excitation and output responses. However, it is usually impossible to directly measure the transfer functions between input excitation and output responses in practical engineering due to variable restrictions and inconveniences. Moreover, researchers mainly focus on studying variety of regularization techniques to improve force identification accuracy. Simple elastic structures, such as beam and plate, are frequently used in numerical simulations. However, vibration isolation systems rather than simple elastic structures are extensively utilized in practical applications. Therefore the force reconstruction problem of vibration isolation system is necessary and worth investigating to provide suggestions for engineers.

In this paper, dynamics of one-stage vibration isolation system is characterized firstly, and then input excitation acting on the system is identified using operational responses measured on rigid body or/and flexible supporting structure theoretically and numerically. Finally, conclusions are drawn from numerical results.

\section{Theoretical Background}

The simplest relation between dynamic responses and excitation can be described as

$$
\mathbf{Y}(\omega)=\mathbf{A}(\omega) \mathbf{F}(\omega)
$$

where $\mathbf{Y} \in R^{m \times 1}$ is vibration response vector and $\mathbf{F} \in R^{n \times 1}$ is excitation force vector. And $\mathbf{A} \in R^{m \times n}$ is frequency response function matrix between excitation and response points. In general, the number of response points $m$ is more than that of excitation points $n$. The least square solution of excitation forces can be obtained by inverse matrix method:

$$
\mathbf{F}=\mathbf{A}^{\dagger} \mathbf{Y}=\left(\mathbf{A}^{H} \mathbf{A}\right)^{-1} \mathbf{A}^{H} \mathbf{Y}
$$

where $\mathbf{A}^{\dagger}$ is Moore-Penrose inverse of matrix $\mathbf{A}$ and superscript $H$ stands for complex conjugate.

The above formula is frequently applied to estimate excitation due to its simplicity. However, it is difficult to directly measure frequency response functions between excitation and response locations in practical engineering. The most common situations are that excitation point is located inside the mechanical equipment and inaccessible. Another common situation is that there is no enough space to arrange sensor or impact hammer on input location. In a word, input excitation cannot be reconstructed by applying only (2) in most practical engineering.

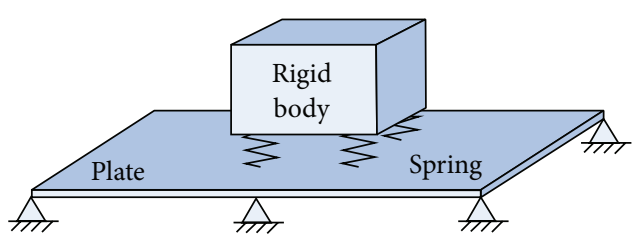

FIGURE 1: Vibration isolation system.

\section{Dynamics of Vibration Isolation System}

3.1. Vibration of Rigid Body. The vibration isolation system, as shown in Figure 1, consists of vibration machine, multiple isolators, and supporting structure. Here the vibration machine is treated as a six-degree-of-freedom rigid body. The origin of coordination system is located at the center of gravity of the rigid body.

The vibration equation of rigid body can be obtained applying Newton's second law:

$$
\mathbf{M} \ddot{\mathbf{u}}^{c}=\mathbf{f}^{m}+\sum_{i=1}^{L} \mathbf{R}_{i}^{c},
$$

where $\mathbf{u}^{c}=\left[\begin{array}{llllll}u_{c} & v_{c} & w_{c} & \theta_{c x} & \theta_{c y} & \theta_{c z}\end{array}\right]^{T}$ is displacement vector (including translational and angular displacements) of gravity center of rigid body and $\mathbf{f}^{m}$ represents excitation force vector acting at CG of rigid body and $\mathbf{R}_{i}^{c}$ is transformed force vector from reaction forces at top end of $i$ th isolator. Moreover, $L$ is total number of isolators.

The mass matrix $\mathbf{M}$ of rigid body is described as

$$
\mathbf{M}=\left[\begin{array}{cccccc}
m & 0 & 0 & 0 & 0 & 0 \\
0 & m & 0 & 0 & 0 & 0 \\
0 & 0 & m & 0 & 0 & 0 \\
0 & 0 & 0 & I_{x x} & -I_{x y} & -I_{x z} \\
0 & 0 & 0 & -I_{y x} & I_{y y} & -I_{y z} \\
0 & 0 & 0 & -I_{z x} & -I_{z y} & I_{z z}
\end{array}\right],
$$

where $m$ is the mass of rigid body and $I_{x x}, I_{x y}, \ldots, I_{z z}$ are the moments of inertia.

As for the isolators, only translational stiffness in $x-, y$-, and $z$-directions is taken into consideration here. And the reaction force vector $\mathbf{R}_{i}^{t}=\left[\begin{array}{lll}F_{x i}^{t} & F_{y i}^{t} & F_{z i}^{t}\end{array}\right]^{T}$ of top end of $i$ th isolator is gained on the basis of Hooke's law:

$$
\mathbf{R}_{i}^{t}=-\mathbf{K}_{i}\left(\mathbf{u}_{i}^{t}-\mathbf{u}_{i}^{b}\right)
$$

where $\mathbf{u}_{i}^{t}=\left[u_{x i}^{t}, u_{y i}^{t}, u_{z i}^{t}\right]^{T}$ and $\mathbf{u}_{i}^{b}=\left[u_{x i}^{b}, u_{y i}^{b}, u_{z i}^{b}\right]^{T}$ are vibration displacement vectors at top and bottom ends of $i$ th isolator, respectively.

The stiffness matrix $\mathbf{K}_{i}$ is defined as

$$
\mathbf{K}_{i}=\mathbf{T}_{i}^{T} \operatorname{diag}\left(k_{i x}, k_{i y}, k_{i z}\right) \mathbf{T}_{i},
$$

where $k_{i x}, k_{i y}$, and $k_{i z}$ are translational stiffness of $i$ th isolator in $x$-, $y$-, and $z$-directions. 
Referring to [23], the transformation matrix $\mathbf{T}_{i}$ is defined as

$$
\mathbf{T}_{i}=\left[\begin{array}{ccc}
\cos \theta_{i} \cos \phi_{i} & \cos \theta_{i} \sin \phi_{i} & -\sin \theta_{i} \\
-\sin \phi_{i} & \cos \phi_{i} & 0 \\
\sin \theta_{i} \cos \phi_{i} & \sin \theta_{i} \cos \phi_{i} & \cos \theta_{i}
\end{array}\right] .
$$

The displacement and reaction force of top end of $i$ th isolator can be transformed to CG of rigid body:

$$
\begin{aligned}
\mathbf{u}_{i}^{t} & =\mathbf{G}_{i} \mathbf{u}^{c}, \\
\mathbf{R}_{i}^{c} & =\mathbf{G}_{i}^{T} \mathbf{R}_{i}^{t} .
\end{aligned}
$$

And transformation matrix $\mathbf{G}_{i}$ is expressed as

$$
\mathbf{G}_{i}=\left[\begin{array}{cccccc}
1 & 0 & 0 & 0 & a_{z}^{i} & -a_{y}^{i} \\
0 & 1 & 0 & -a_{z}^{i} & 0 & a_{x}^{i} \\
0 & 0 & 1 & a_{y}^{i} & -a_{x}^{i} & 0
\end{array}\right]
$$

where $a_{x}^{i}, a_{y}^{i}$, and $a_{z}^{i}$ are projections onto $x$-, $y$-, and $z$-axis of the distances from CG of rigid body to the top end of $i$ th isolator.

3.2. Vibration of Flexible Supporting Structure. Flexible supporting structure is assumed as a simply supported plate in this paper. The reaction force vector $\mathbf{R}_{i}^{b}=\left[\begin{array}{lll}F_{x i}^{b} & F_{y i}^{b} & F_{z i}^{b}\end{array}\right]^{T}$ acting on the plate is

$$
\mathbf{R}_{i}^{b}=-\mathbf{R}_{i}^{t}
$$

Here, plainly, vibrations of the plate are ignored. Plainly, reaction forces $F_{x i}^{b}$ and $F_{y i}^{b}$ are modeled as moments about $y$ and $x$-directions acting on the flexible plate. So the transverse displacement $W(\sigma, \omega)$ can be described as the following equation:

$$
\begin{aligned}
& D \nabla^{4} W+\rho h \frac{\partial^{2} W}{\partial t^{2}}=\sum_{i=1}^{L}\left(F_{z i}^{b} \delta\left(\sigma-\sigma_{i}\right)\right. \\
& \left.-\frac{\partial\left(M_{x i}^{b} \delta\left(\sigma-\sigma_{i}\right)\right)}{\partial y}+\frac{\partial\left(M_{y i}^{b} \delta\left(\sigma-\sigma_{i}\right)\right)}{\partial x}\right),
\end{aligned}
$$

where $h, \rho, E, \mu$ are thickness, density, Young's modulus, and Poisson's ratio of the supporting plate, respectively. $D=$ $E h^{3} /\left[12\left(1-\mu^{2}\right)\right]$ is bending stiffness of the plate. And $\sigma_{i}=$ $\left(x_{b i}, y_{b i}\right)$ stands for $i$ th isolator location on the plate.

Ignoring the effect of plate thickness, the moments $M_{x i}^{b}$ and $M_{y i}^{b}$ are

$$
\begin{aligned}
& M_{x i}^{b}=-F_{y i}^{b} l, \\
& M_{y i}^{b}=F_{x i}^{b} l,
\end{aligned}
$$

where $l$ is the height of isolator.
The transverse vibration displacement $W(\sigma, \omega)$ can be obtained according to modal superposition principle by mode shape vector $\boldsymbol{\Phi}=\left[\begin{array}{llll}\psi_{1}(\sigma) & \psi_{2}(\sigma) & \cdots & \psi_{p}(\sigma)\end{array}\right]$ and coefficient matrix $\boldsymbol{\alpha}=\left[\begin{array}{llll}\alpha_{1} & \alpha_{2} & \cdots & \alpha_{p}\end{array}\right]^{T}$ :

$$
W(\sigma, \omega)=\boldsymbol{\Phi} \boldsymbol{\alpha} \text {. }
$$

Submitting (12) and (13) into (11) and applying modal orthogonality, vibration motion associated with $I$ th $(I=$ $1,2, \ldots, p)$ mode can be written as [24]

$$
\begin{aligned}
& M_{I}\left(\omega_{I}^{2}-\omega^{2}\right) \alpha_{I} \\
& \quad=\sum_{i=1}^{L}\left(l \frac{\partial \psi_{I}\left(\sigma_{i}\right)}{\partial x} l \frac{\partial \psi_{I}\left(\sigma_{i}\right)}{\partial y} \psi_{I}\left(\sigma_{i}\right)\right) \mathbf{R}_{i}^{b},
\end{aligned}
$$

where the modal mass is

$$
M_{I}=\int \rho h \psi_{I}^{2}(\sigma) d \sigma=\frac{1}{4} \rho h L_{x} L_{y} .
$$

Taking the structural damping into account, the following equation can be obtained:

$$
\begin{aligned}
& M_{I}\left(\omega_{I}^{2}+j \eta_{I} \omega_{I}^{2}-\omega^{2}\right) \alpha_{I} \\
& \quad=\sum_{i=1}^{L}\left(l \frac{\partial \psi_{I}\left(\sigma_{i}\right)}{\partial x} l \frac{\partial \psi_{I}\left(\sigma_{i}\right)}{\partial y} \psi_{I}\left(\sigma_{i}\right)\right) \mathbf{R}_{i}^{b},
\end{aligned}
$$

where $\eta_{I}$ is the loss factor of $I$ th mode. For simplicity, the above $p$ simultaneous equations can be converted into the following matrix form:

$$
-\omega^{2} \mathbf{M}_{s} \boldsymbol{\alpha}+\mathbf{K}_{s} \boldsymbol{\alpha}=\sum_{i=1}^{L} \mathbf{B}_{i} \mathbf{C}_{1} \mathbf{R}_{i}^{b} .
$$

The matrices $\mathbf{M}_{s}$ and $\mathbf{K}_{s}$ are defined as

$$
\begin{aligned}
\mathbf{M}_{s} & =\operatorname{diag}\left(M_{I}, M_{I}, \ldots, M_{I}\right), \\
\mathbf{K}_{s} & =M_{I} \operatorname{diag}\left(\omega_{1}^{2}\left(1+j \eta_{1}\right), \omega_{2}^{2}\left(1+j \eta_{2}\right), \ldots,\right. \\
& \left.\omega_{p}{ }^{2}\left(1+j \eta_{p}\right)\right) .
\end{aligned}
$$

The force location matrix and constant matrix are, respectively,

$$
\begin{aligned}
\mathbf{B}_{i}=\left[\begin{array}{ccc}
\psi_{1 x}\left(\sigma_{i}\right) & \psi_{1 y}\left(\sigma_{i}\right) & \psi_{1}\left(\sigma_{i}\right) \\
\psi_{2 x}\left(\sigma_{i}\right) & \psi_{2 y}\left(\sigma_{i}\right) & \psi_{2}\left(\sigma_{i}\right) \\
\vdots & \vdots & \vdots \\
\psi_{p x}\left(\sigma_{i}\right) & \psi_{p y}\left(\sigma_{i}\right) & \psi_{p}\left(\sigma_{i}\right)
\end{array}\right], \\
\mathbf{C}_{1}=\operatorname{diag}(l, l, 1) .
\end{aligned}
$$

For a simply supported plate of dimensions $L_{x}$ and $L_{y}$, Ith mode frequency and mode shape function are

$$
\begin{aligned}
\omega_{I} & =\sqrt{\left[\left(\frac{m \pi}{L_{x}}\right)^{2}+\left(\frac{n \pi}{L_{y}}\right)^{2}\right]^{2} \frac{D}{\rho h}}, \\
\psi_{I}(\sigma) & =\psi_{m, n}(x, y)=\sin \left(\frac{m \pi x}{L_{x}}\right) \sin \left(\frac{n \pi y}{L_{y}}\right) .
\end{aligned}
$$


So the functions $\psi_{I x}\left(\sigma_{i}\right)$ and $\psi_{I y}\left(\sigma_{i}\right)$ are

$$
\begin{aligned}
\psi_{I x}\left(\sigma_{i}\right) & =\frac{\partial \psi_{m, n}\left(x_{b i}, y_{b i}\right)}{\partial x} \\
& =\frac{m \pi}{L_{x}} \cos \left(\frac{m \pi x_{b i}}{L_{x}}\right) \sin \left(\frac{n \pi y_{b i}}{L_{y}}\right), \\
\psi_{I y}\left(\sigma_{i}\right) & =\frac{\partial \psi_{m, n}\left(x_{b i}, y_{b i}\right)}{\partial y} \\
& =\frac{n \pi}{L_{y}} \sin \left(\frac{m \pi x_{b i}}{L_{x}}\right) \cos \left(\frac{n \pi y_{b i}}{L_{y}}\right),
\end{aligned}
$$

where $m$ and $n$ are integers representing $I$ th plate mode.

The displacement vector at bottom end of $i$ th isolator is

$$
\mathbf{u}_{i}^{b}=\left[\begin{array}{lll}
0 & 0 & W\left(\sigma_{i}\right)
\end{array}\right]^{T}=\mathbf{C}_{2} \mathbf{B}_{i}^{T} \boldsymbol{\alpha},
$$

where the constant matrix is

$$
\mathbf{C}_{2}=\operatorname{diag}(0,0,1) \text {. }
$$

3.3. Coupling Vibration of Vibration Isolation System. The coupling vibration of the whole vibration isolation system can be obtained by combining the vibration of rigid body with that of plate:

$$
\left[\begin{array}{cc}
\mathbf{K}^{m m} & \mathbf{K}^{m s} \\
\mathbf{K}^{s m} & \mathbf{K}^{s s}
\end{array}\right]\left\{\begin{array}{l}
\mathbf{u}^{c} \\
\boldsymbol{\alpha}
\end{array}\right\}-\omega^{2}\left[\begin{array}{cc}
\mathbf{M} & \mathbf{0} \\
\mathbf{0} & \mathbf{M}_{s}
\end{array}\right]\left\{\begin{array}{l}
\mathbf{u}^{c} \\
\boldsymbol{\alpha}
\end{array}\right\}=\left\{\begin{array}{c}
\mathbf{f}^{m} \\
\mathbf{0}
\end{array}\right\},
$$

where submatrices $\mathbf{K}^{m m}, \mathbf{K}^{m s}, \mathbf{K}^{s m}$, and $\mathbf{K}^{s s}$ are expressed as

$$
\begin{aligned}
\mathbf{K}^{m m} & =\sum_{i=1}^{L} \mathbf{G}_{i}^{T} \mathbf{K}_{i} \mathbf{G}_{i}, \\
\mathbf{K}^{m s} & =-\sum_{i=1}^{L} \mathbf{G}_{i}^{T} \mathbf{K}_{i} \mathbf{C}_{2} \mathbf{B}_{i}{ }^{T}, \\
\mathbf{K}^{s m} & =-\sum_{i=1}^{L} \mathbf{B}_{i} \mathbf{C}_{1} \mathbf{K}_{i} \mathbf{G}_{i}, \\
\mathbf{K}^{s s} & =\mathbf{K}_{s}+\sum_{i=1}^{L} \mathbf{B}_{i} \mathbf{C}_{1} \mathbf{K}_{i} \mathbf{C}_{2} \mathbf{B}_{i}{ }^{T} .
\end{aligned}
$$

\section{Identification of Excitation Forces Acting on Mechanical Engine in Suit}

4.1. Force Identification Based on Measured Responses of Rigid Body. The excitation forces can be derived through the vibration equation of the isolation system:

$$
\mathbf{f}^{m}=\left[\left(\mathbf{K}^{m m}-\omega^{2} \mathbf{M}\right)+\mathbf{K}^{m s}\left(\omega^{2} \mathbf{M}_{s}-\mathbf{K}^{s s}\right)^{-1} \mathbf{K}^{s m}\right] \mathbf{u}^{c}
$$

The dynamic vibration of simply supported plate is characterized by modal parameters in unloaded condition in (26). Another attractive method is directly measuring transfer functions of the plate. The dynamic displacement vector at bottom ends of all the isolators under operational condition is

$$
\mathbf{u}^{b}=\mathbf{Q R}^{b}
$$

where

$$
\begin{gathered}
\mathbf{u}^{b}=\left\{\begin{array}{c}
\mathbf{u}_{1}^{b} \\
\vdots \\
\mathbf{u}_{L}^{b}
\end{array}\right\}, \\
\mathbf{R}^{b}=\left\{\begin{array}{c}
\mathbf{R}_{1}^{b} \\
\vdots \\
\mathbf{R}_{L}^{b}
\end{array}\right\} .
\end{gathered}
$$

And $\mathbf{Q}$ is transfer function matrix between isolator locations on the plate without the attachment of the rigid body.

The reaction force vector of all the isolators can be expressed as

$$
\mathbf{R}^{b}=\mathbf{K}\left(\mathbf{u}^{t}-\mathbf{u}^{b}\right)
$$

where

$$
\begin{aligned}
& \mathbf{K}=\left[\begin{array}{lll}
\mathbf{K}_{1} & & \\
& \ddots & \\
& & \mathbf{K}_{L}
\end{array}\right], \\
& \mathbf{u}^{t}=\left\{\begin{array}{c}
\mathbf{u}_{1}^{t} \\
\vdots \\
\mathbf{u}_{L}^{t}
\end{array}\right\} .
\end{aligned}
$$

Submitting (27) into (29) yields the following formula:

$$
\mathbf{R}^{b}=(\mathbf{I}+\mathbf{K Q})^{-1} \mathbf{K} \mathbf{u}^{t} .
$$

The relationship between the displacement vector $\mathbf{u}_{i}^{b}$ of $i$ th isolator and the displacement vector $\mathbf{u}^{b}$ of all the isolators is

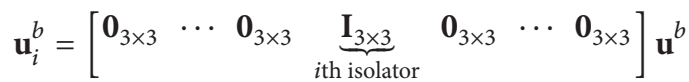

$$
\begin{aligned}
& =\mathbf{S}_{i} \mathbf{u}^{b} \text {. }
\end{aligned}
$$

Similarly, the following equations can be obtained:

$$
\begin{aligned}
\mathbf{u}_{i}^{t} & =\mathbf{S}_{\mathbf{i}} \mathbf{u}^{t}, \\
\mathbf{u}^{t} & =\sum_{i=1}^{L} \mathbf{S}_{i}{ }^{T} \mathbf{u}_{i}^{t}, \\
\mathbf{R}_{i}^{b} & =\mathbf{S}_{i} \mathbf{R}^{b}
\end{aligned}
$$


Submitting (8) into (31), the reaction forces of all isolators can be expressed as the function of displacements at CG of rigid body:

$$
\mathbf{R}^{b}=(\mathbf{I}+\mathbf{K Q})^{-1} \mathbf{K}\left(\sum_{i=1}^{L} \mathbf{S}_{i}^{T} \mathbf{G}_{i}\right) \mathbf{u}^{c} .
$$

The excitation forces can be derived as the following expression based on (3) and (10):

$$
\mathbf{f}^{m}=-\omega^{2} \mathbf{M} \mathbf{u}^{c}+\left(\sum_{i=1}^{L} \mathbf{G}_{i}^{T} \mathbf{S}_{i}\right) \mathbf{R}^{b} .
$$

Submitting (34) into (35), the excitation forces can be extracted by the vibration displacements at CG of rigid body:

$$
\mathbf{f}^{m}=\mathbf{H}_{c} \mathbf{u}^{c},
$$

where

$$
\begin{aligned}
\mathbf{H}_{c} & =\left[-\omega^{2} \mathbf{M}\right. \\
& \left.+\left(\sum_{i=1}^{L} \mathbf{G}_{i}^{T} \mathbf{S}_{i}\right)(\mathbf{I}+\mathbf{K Q})^{-1} \mathbf{K}\left(\sum_{i=1}^{L} \mathbf{S}_{i}^{T} \mathbf{G}_{i}\right)\right] .
\end{aligned}
$$

Theoretically speaking, (26) and (36) use modal parameters and transfer functions to characterize the dynamic performance of plate, respectively. And the latter one is more suitable for practical applications because of easier and simpler measurements.

The measured displacement vector of CG of rigid body is employed in (36) to determine input excitation. However, there is no way to measure directly the displacements of CG. So it is necessary to determine the displacements at CG of rigid body through the vibration displacements at accessible response points on the surface of rigid body and the plate under operational condition and then to extract the excitation forces.

The displacements measured on the surface of rigid body and that of CG of rigid body have the following relationship:

$$
\mathbf{u}_{r}=\left\{\begin{array}{c}
\mathbf{u}_{r 1} \\
\vdots \\
\mathbf{u}_{r j} \\
\vdots \\
\mathbf{u}_{r J}
\end{array}\right\}=\left[\begin{array}{c}
\mathbf{G}_{r 1} \\
\vdots \\
\mathbf{G}_{r j} \\
\vdots \\
\mathbf{G}_{r J}
\end{array}\right] \mathbf{u}^{c}=\mathbf{G}_{r c} \mathbf{u}^{c} \quad(j=1,2, \ldots, J),
$$

where the variable $J$ denotes the number of response points on the surface of rigid body. Note that at least three response points should be measured in order to get least square solution of displacement vector of the gravity center.

It is worth mentioning that the above equation is underdetermined with infinite solutions when only one response point is applied to determine excitation forces. In addition, use of two response points for force identification is feasible theoretically and unique solution can be obtained. Practically, it is highly likely that the matrix $\mathbf{G}_{r c}$ is rank deficient and its inversion will lead to wrong estimation result. Therefore, more than two points are supposed to be selected for this force identification problem.

Combining (38) with (36), the external excitation forces can be extracted by the vibration displacements on the surface of rigid body:

$$
\mathbf{f}^{m}=\mathbf{H}_{c} \mathbf{G}_{r c}^{\dagger} \mathbf{u}_{r}
$$

4.2. Force Identification Based on Measured Responses of Flexible Plate. In some special situations, it is impossible to carry out vibration measurement on the surface of the engine. For example, the operating engine is too hot to arrange vibration sensors on its surface. In such a situation, only dynamic responses on supporting structure can be measured to determine excitation forces.

The following relationship between measured displacements of the plate and reaction forces of bottom end of isolators can be gained:

$$
\mathbf{u}_{s}=\mathbf{H R}^{b}
$$

Combining (40) with (34), it follows that

$$
\mathbf{u}_{s}=\mathbf{H}_{s c} \mathbf{u}^{c},
$$

where

$$
\mathbf{H}_{s c}=\mathbf{H}(\mathbf{I}+\mathbf{K Q})^{-1} \mathbf{K}\left(\sum_{i=1}^{L} \mathbf{S}_{i}^{T} \mathbf{G}_{i}\right),
$$

where $\mathbf{H}$ is transfer function matrix for the supporting plate without the rigid body loaded.

In order to ensure the uniqueness of the solution, at least six response points should be allocated on the flexible plate when computing the displacements at CG of rigid body on the basis of (41). Then the external excitation can be determined by the displacements of the plate:

$$
\mathbf{f}^{m}=\mathbf{H}_{c} \mathbf{H}_{s c}^{\dagger} \mathbf{u}_{s} \text {. }
$$

4.3. Force Identification Based on Measured Responses of Rigid Body and Flexible Plate. The following relationship can be achieved according to (38) and (41):

$$
\mathbf{u}_{r s}=\left\{\begin{array}{l}
\mathbf{u}_{r} \\
\mathbf{u}_{s}
\end{array}\right\}=\left[\begin{array}{l}
\mathbf{G}_{r c} \\
\mathbf{H}_{s c}
\end{array}\right] \mathbf{u}^{c}=\mathbf{H}_{r s c} \mathbf{u}^{c} .
$$

It is worth noting that the row number of displacement vector $\mathbf{u}_{r_{s}}$ should be equivalent to or more than six. For example, three translational displacements of one point on the surface of rigid body are measured, and then at least three points on the plate should be used. The excitation force acting at CG of rigid body can be reconstructed through dynamic responses on both rigid body and plate:

$$
\mathbf{f}^{m}=\mathbf{H}_{c} \mathbf{H}_{r s c}^{\dagger}{ }^{\dagger} \mathbf{u}_{r s} .
$$


TABLE 1: Geometric and material parameters of the vibration isolation system.

\begin{tabular}{|c|c|c|c|c|}
\hline System component & Geometric parameters & & Material parameters & \\
\hline Rigid body & $\begin{array}{l}\text { Length }(\mathrm{mm}) \\
\text { Width }(\mathrm{mm}) \\
\text { Height }(\mathrm{mm})\end{array}$ & $\begin{array}{c}350 \\
120 \\
50\end{array}$ & Density $\left(\mathrm{kg} / \mathrm{m}^{3}\right)$ & 7800 \\
\hline Plate & $\begin{array}{l}\text { Length }(\mathrm{mm}) \\
\text { Width }(\mathrm{mm}) \\
\text { Height }(\mathrm{mm})\end{array}$ & $\begin{array}{c}800 \\
800 \\
5\end{array}$ & $\begin{array}{c}\text { Young's modulus }(\mathrm{Pa}) \\
\text { Poisson's ratio } \\
\text { Loss factor } \\
\text { Density }\left(\mathrm{kg} / \mathrm{m}^{3}\right)\end{array}$ & $\begin{array}{c}2.16 e 11 \\
0.3 \\
0.01 \\
7800\end{array}$ \\
\hline
\end{tabular}

TABLE 2: Position coordinates of the resilient isolators ( $\mathrm{mm})$.

\begin{tabular}{lcc}
\hline Number of isolators Top end of isolators & Bottom end of isolators \\
\hline 1 & $(-165,-50,-25)$ & $(235,350,2.5)$ \\
2 & $(165,-50,-25)$ & $(565,350,2.5)$ \\
3 & $(165,50,-25)$ & $(565,450,2.5)$ \\
4 & $(-165,50,-25)$ & $(235,450,2.5)$ \\
\hline
\end{tabular}

TABLE 3: Locations of response points ( $\mathrm{mm}$ ).

\begin{tabular}{llll}
\hline Rigid body & \multicolumn{3}{c}{ Flexible supporting plate } \\
\hline & $(250,100,2.5)$ & $(700,350,2.5)$ & $(200,400,2.5)$ \\
$(100,10,25)$ & $(400,600,2.5)$ & $(100,700,2.5)$ & $(500,750,2.5)$ \\
$(20,60,-10)$ & $(650,200,2.5)$ & $(600,500,2.5)$ & $(300,400,2.5)$ \\
$(-175,20,-15)$ & $(700,550,2.5)$ & $(750,300,2.5)$ & $(400,350,2.5)$ \\
& $(450,200,2.5)$ & $(500,300,2.5)$ & \\
\hline
\end{tabular}

\section{Results and Discussions}

The proposed vibration isolation system is employed in this section to determine the excitation forces acting at gravity center of rigid body by means of measured displacements.

The geometric and material parameters of the vibration isolation system as shown in Figure 2 are listed in Table 1. Rigid body is connected with the simply supported plate through four identical resilient isolators with stiffness coefficients $53358 \mathrm{~N} / \mathrm{m}, 53358 \mathrm{~N} / \mathrm{m}$, and $99535 \mathrm{~N} / \mathrm{m}$ in $x-, y-$, and $z$-directions, respectively. And loss factor of the isolators is 0.1 . The position coordinates of resilient isolators are shown in Table 2.

A frequency range of $0-200 \mathrm{~Hz}$ is selected to analyze the vibration source features of rigid body. The locations of response points allocated on surface of rigid body and supporting plate are present in Table 3. As for the third method, the first two points on the rigid body and the first, third, and fifth points on the plate are employed to identify the inputs.

The excitation forces acting at CG of rigid body are assumed as a column vector $[1,1,1,1,1,1]^{T}$. In order to investigate the effect of practical environment on measurement accuracy, some random noises are added into the actual displacement responses to produce measured responses. Comparisons between actual and measured displacements in $x$-, $y$-, and $z$-directions of first response point on rigid body surface are shown in Figure 3.

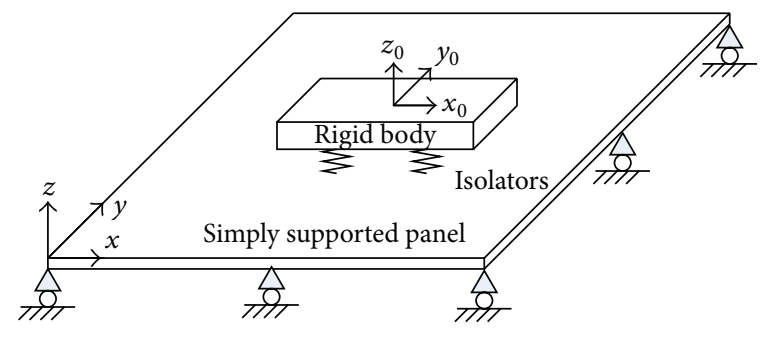

FIGURE 2: Schematic of vibration isolation system.

Figure 4 displays comparison between actual and measured transverse displacements of first point listed in Table 3 on the plate.

The estimated excitation forces are present in Figure 5. Three different methods based on (39), (43), and (45) are adopted to determine input excitation acting at CG of rigid body. The first method uses measured responses on rigid body surface, second method responses on the supporting plate, and third method responses on both rigid body surface and the plate.

As shown in Figure 5, the first method shows most accurate estimation inputs among these three methods. The second method, applying measured responses only on the plate, gives the worst estimation results, especially forces in $x$ - and $y$-directions. On one hand, SNR of vibration responses on the plate is lower than those on rigid body. On the other hand, condition number of matrix $\mathbf{H}_{s c}$ is much large due to similar vibration characteristics between CG and different points on the plate. As a result, the errors in responses are amplified significantly and reconstructed forces are distorted seriously. One can improve effectively estimation accuracy by adding responses measured on rigid body into those measured on plate.

An average error estimate for each excitation component and total average error are used here, given as follows:

$$
\begin{aligned}
\mathrm{AE}_{j} & =\left(\frac{1}{N} \sum_{i=1}^{N}\left|20 \log \frac{\widehat{F}_{j, i}}{F_{j, i}}\right|_{j}^{2}\right)^{1 / 2}, \\
\mathrm{TAE} & =\frac{1}{n} \sum_{j=1}^{n} \mathrm{AE}_{j},
\end{aligned}
$$

where $\mathrm{AE}_{j}$ is the average error for $j$ th excitation in $\mathrm{dB}$ and TAE is total average error in $\mathrm{dB}$. $N$ is the number of frequency 
TABLE 4: Average error in $\mathrm{dB}$ calculated in the frequency range of $0-200 \mathrm{~Hz}$.

\begin{tabular}{|c|c|c|c|c|c|c|c|}
\hline & $F_{x}$ & $F_{y}$ & $F_{z}$ & $M_{x}$ & $M_{y}$ & $M_{z}$ & TAE \\
\hline First method & 2.2816 & 4.1058 & 4.5891 & 0.4457 & 0.5063 & 0.8213 & 2.1250 \\
\hline Second method & 13.2619 & 32.3192 & 3.8388 & 6.0782 & 0.4154 & 0.9810 & 9.4824 \\
\hline Third method & 3.5047 & 5.4517 & 5.6535 & 0.3752 & 1.3076 & 1.7051 & 2.9996 \\
\hline
\end{tabular}

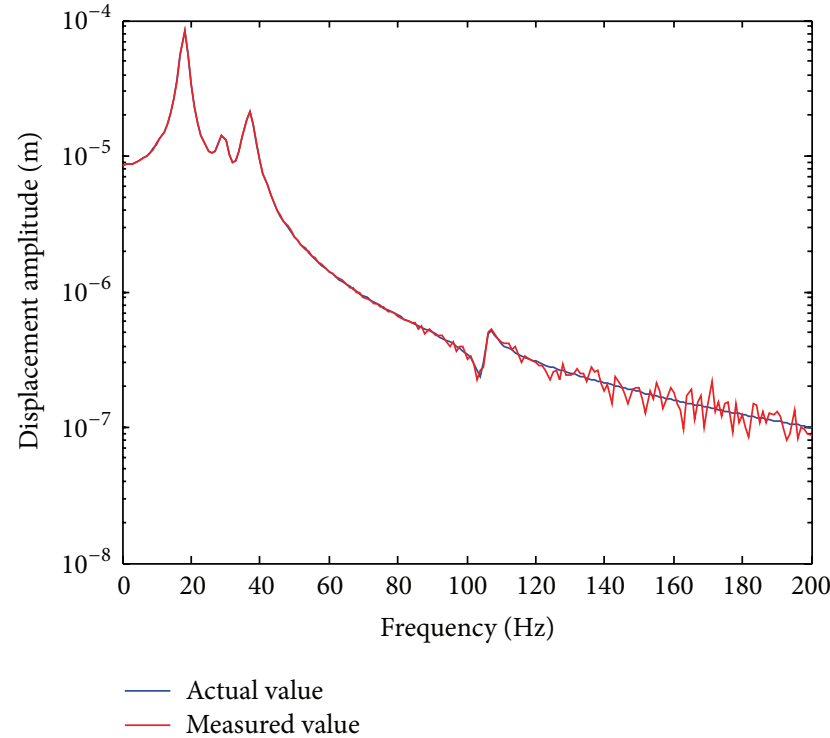

(a)

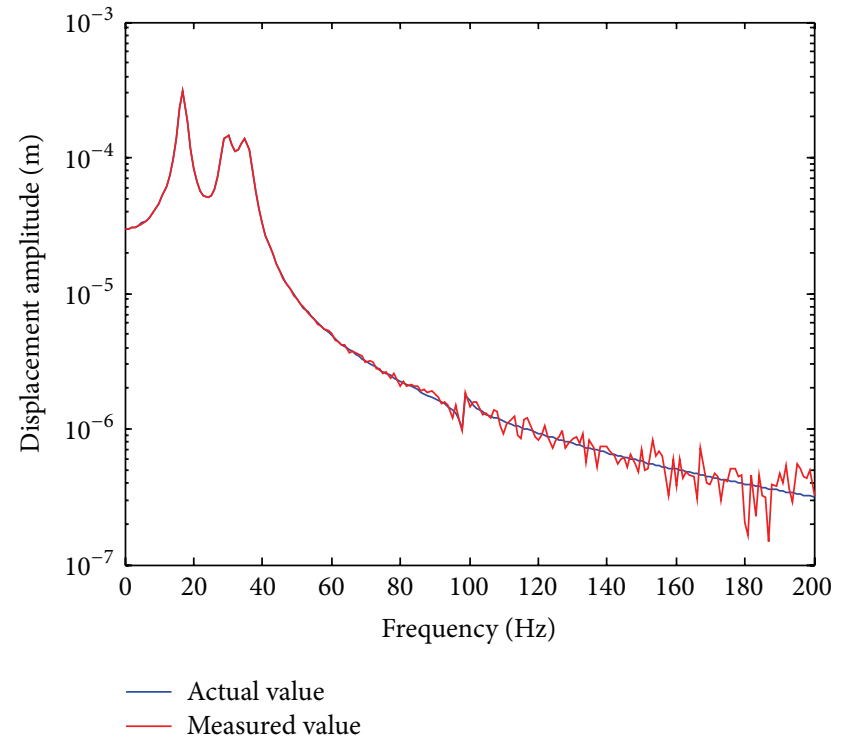

(b)

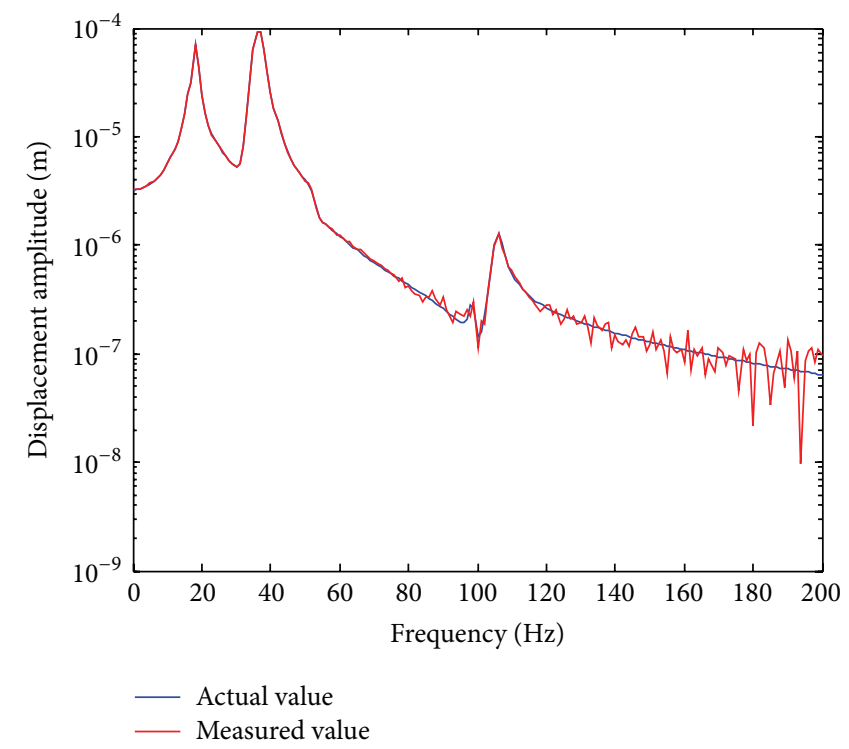

(c)

FIGURE 3: Comparison of actual and measured displacements of first point on the surface of rigid body. (a) Displacement in $x$-direction. (b) Displacement in $y$-direction. (c) Displacement in $z$-direction.

bands in frequency range, $\widehat{F}_{j, i}$ and $F_{j, i}$ are the reconstructed and actual $j$ th excitation forces, and $n$ is the number of excitation components.

Table 4 shows average error for each excitation component and total average error obtained by input estimations reconstructed by dynamic responses measured on rigid body and flexible plate.
The transfer function matrix of the second method is ill-conditioned, and the small errors in measured responses are enlarged through inversion of transfer function matrix. So far, plenty of regularization methods, such as Tikhonov regularization method, Truncated Singular Value Decomposition, and iterative method, are widely utilized for input determination. Here, attention is paid to decreasing the 


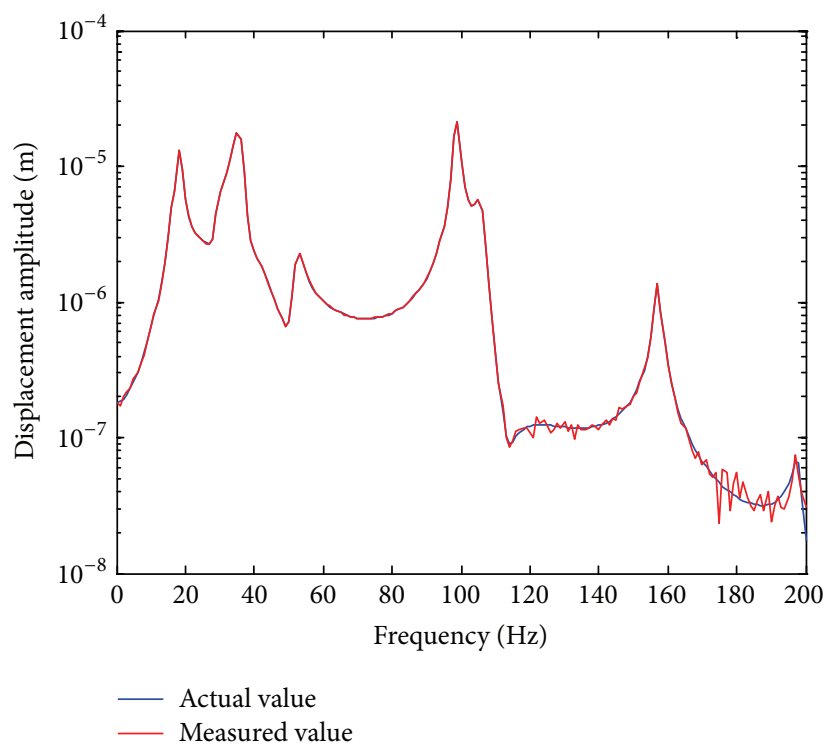

FIGURE 4: Comparison of actual and measured displacements in $z$-direction of first point on the plate.

TABLE 5: Average error in $\mathrm{dB}$ calculated in the frequency range of $0-200 \mathrm{~Hz}$.

\begin{tabular}{|c|c|c|c|c|c|c|c|}
\hline & $F_{x}$ & $F_{y}$ & $F_{z}$ & $M_{x}$ & $M_{y}$ & $M_{z}$ & TAE \\
\hline Improved second method & 3.4327 & 21.8904 & 1.0970 & 2.6124 & 0.0365 & 4.8571 & 5.6544 \\
\hline
\end{tabular}

noise included in measured response rather than the abovementioned regularization technologies.

According to the measurements of frequency response function (auto-spectrum and cross-spectrum between input and output), a similar method is used to decrease the measurement noise. Equation (40) can be transformed into

$$
\mathbf{H}^{H} \mathbf{u}_{s}=\mathbf{H}^{H} \mathbf{H R}^{b} .
$$

Define auto-power spectral density function as

$$
\mathbf{S}_{H H}(\omega)=E\left\{\mathbf{H}(\omega)^{H} \mathbf{H}(\omega)\right\},
$$

and define cross-power spectral density function as

$$
\mathbf{S}_{H u}(\omega)=E\left\{\mathbf{H}(\omega)^{H} \mathbf{u}_{s}(\omega)\right\} \text {. }
$$

Then force vector $\mathbf{R}^{b}$ can be derived:

$$
\mathbf{R}^{b}=\mathbf{S}_{H H}{ }^{-1} \mathbf{S}_{H u} .
$$

So the input excitation applied to rigid body can be determined by dynamic responses measured on plate through combination of (34), (35), and (50):

$$
\begin{aligned}
\mathbf{f}^{m} & =\left[-\omega^{2} \mathbf{M}\left(\mathbf{K}\left(\sum_{i=1}^{L} \mathbf{S}_{i}^{T} \mathbf{G}_{i}\right)\right)^{-1}(\mathbf{I}+\mathbf{K Q})\right. \\
& \left.+\left(\sum_{i=1}^{L} \mathbf{G}_{i}^{T} \mathbf{S}_{i}\right)\right] \mathbf{S}_{H H}{ }^{-1} \mathbf{S}_{H u} .
\end{aligned}
$$

The number of averages in estimating $\mathbf{S}_{H H}$ and $\mathbf{S}_{H u}$ is 500. The calculation results of input excitation extracted by responses measured on the plate are shown in Figure 6 (improved result is determined by (51) and original result by (43)). The estimation results represent a certain degree of improvement on accuracy of input estimations since (51) is applied. And corresponding average errors for input estimations by improved method are listed in Table 5 .

Another case investigated here is excitation force vector $[0,0,1,1,1,0]^{T}$ acting at CG of rigid body. The reconstructed excitation is illustrated in Figure 7. The second method adopting responses only on the plate shows the worst estimation results. However, using responses measured on rigid body can effectively improve the estimation accuracy.

It is worth mentioning that estimation results previously obtained distorted seriously at natural frequencies associated with vibration of rigid body. Regularization technologies should be employed to improve estimation accuracy above these natural frequencies.

\section{Conclusions}

Input excitation of vibration isolation system is reconstructed by assuming dynamic responses on rigid body and supporting structures are known from in-suit measurements. Estimation results of input excitation suggest that responses on rigid body are likely to provide a more accurate input estimation than responses on supporting structure. So the dynamic responses on engine are encouraged to be measured in engineering applications to extract inputs acting on vibration isolation systems. Moreover, with respect to responses on supporting structure employed to identify input 

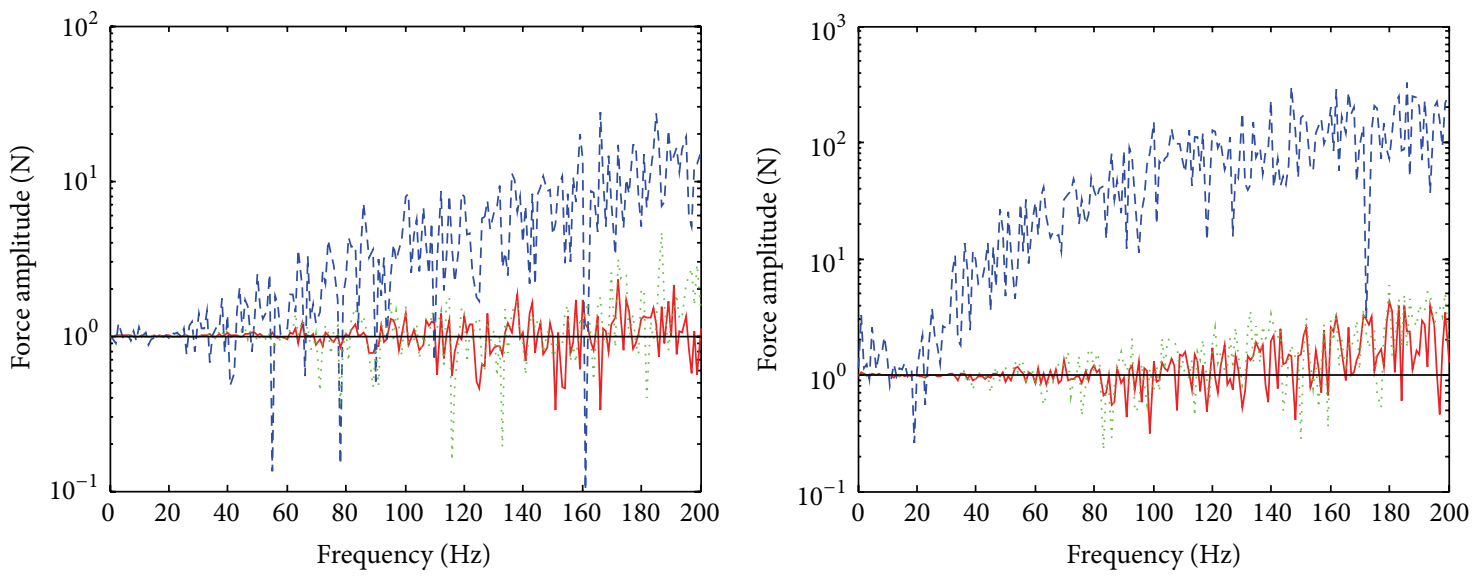

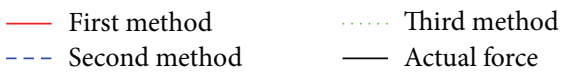

(a)

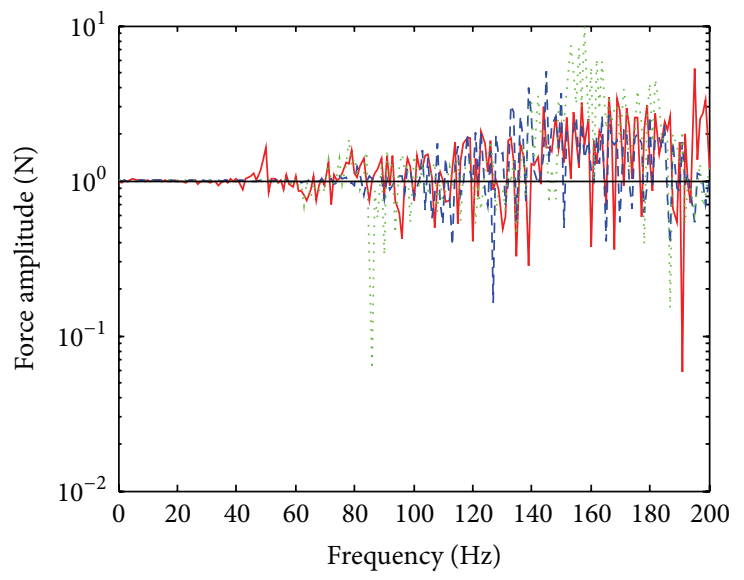

- First method

- - - Second method

(c)

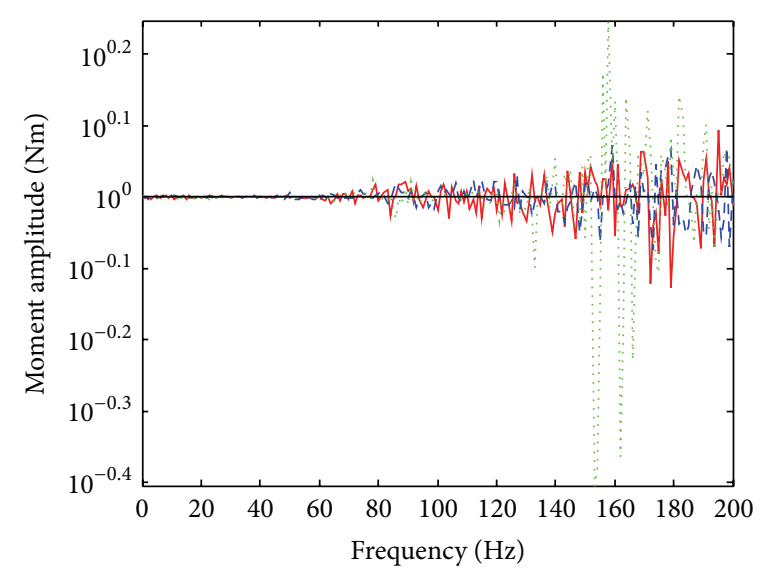

$\begin{array}{ll}\text { - First method } & \ldots . . \text { Third method } \\ \text { - }- \text { Second method } & \quad \text { Actual moment }\end{array}$

(e)

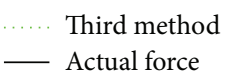

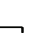$$
0
$$

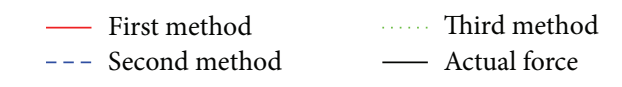

(b)

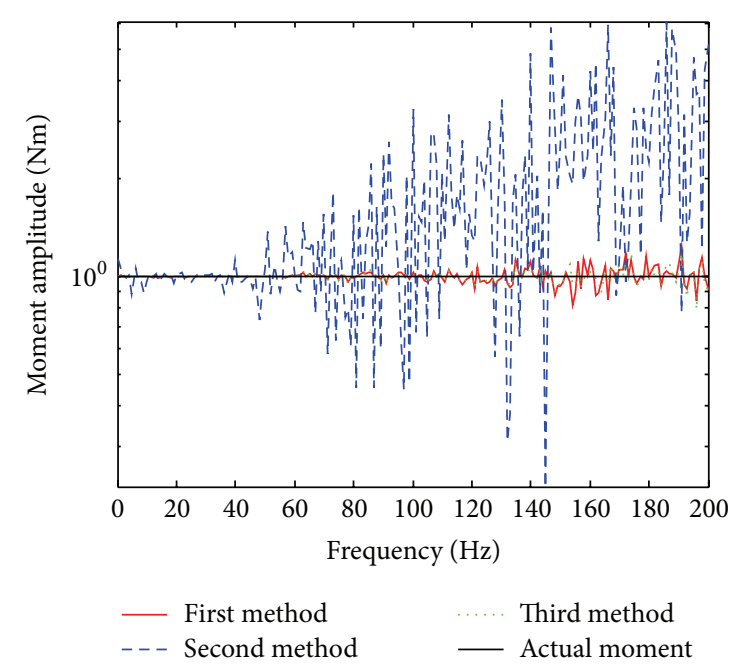

(d)

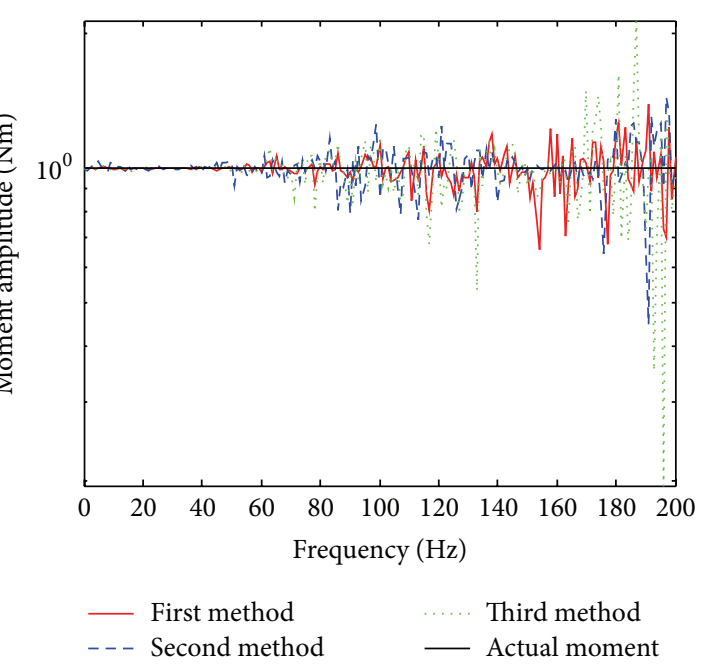

(f)

Figure 5: Comparison of estimated inputs by three different methods and actual inputs. (a) Force in $x$-direction. (b) Force in $y$-direction. (c) Force in $z$-direction. (d) Moment around $x$-direction. (e) Moment around $y$-direction. (f) Moment around $z$-direction. 

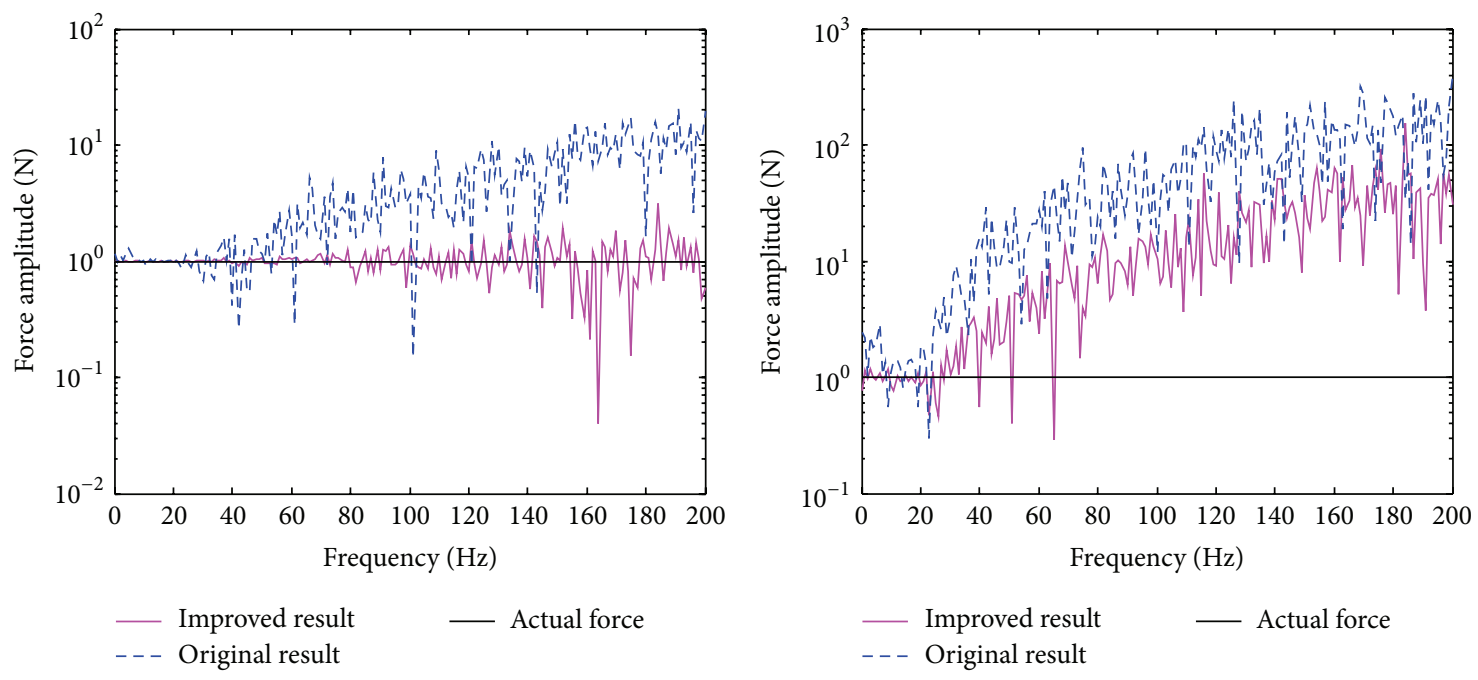

(a)

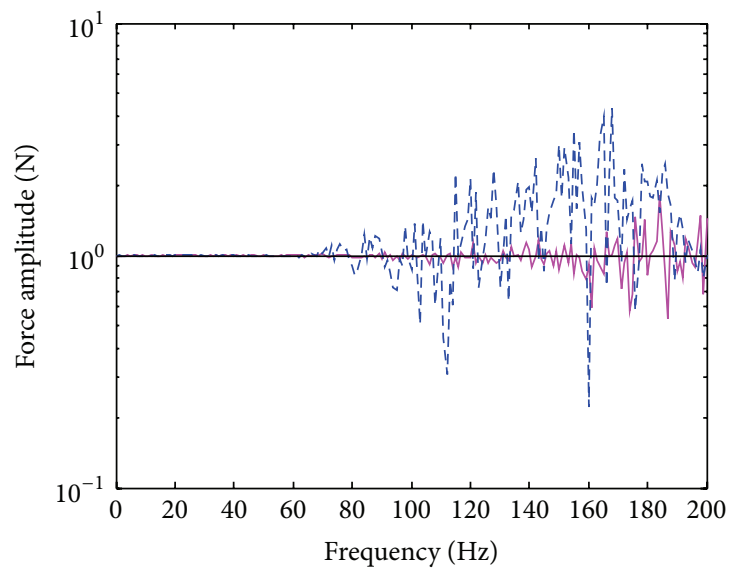

(b)

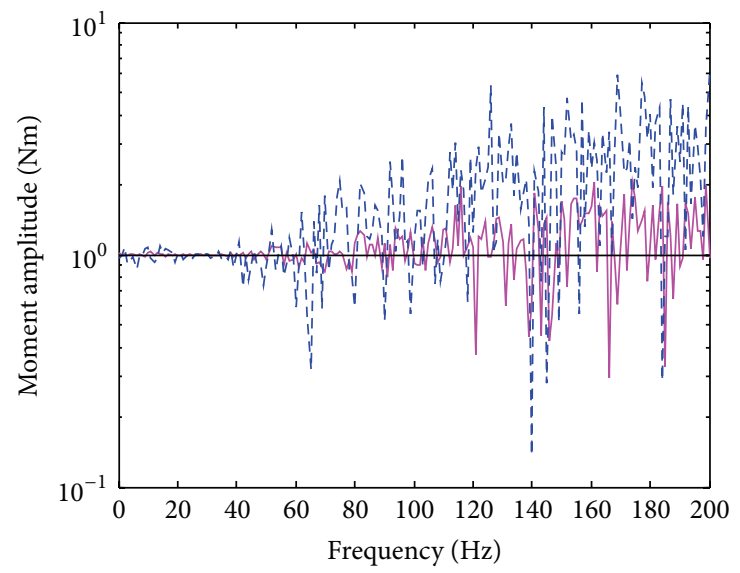

$\begin{array}{ll}\text { - Improved result } & \text { - Actual force } \\ --- & \text { Original result }\end{array}$

(c)

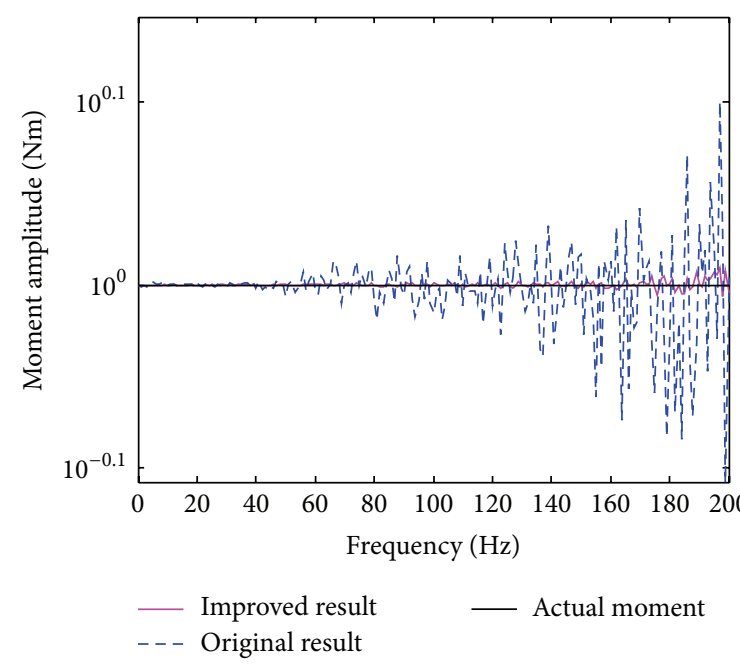

(e)

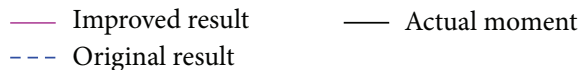

(d)

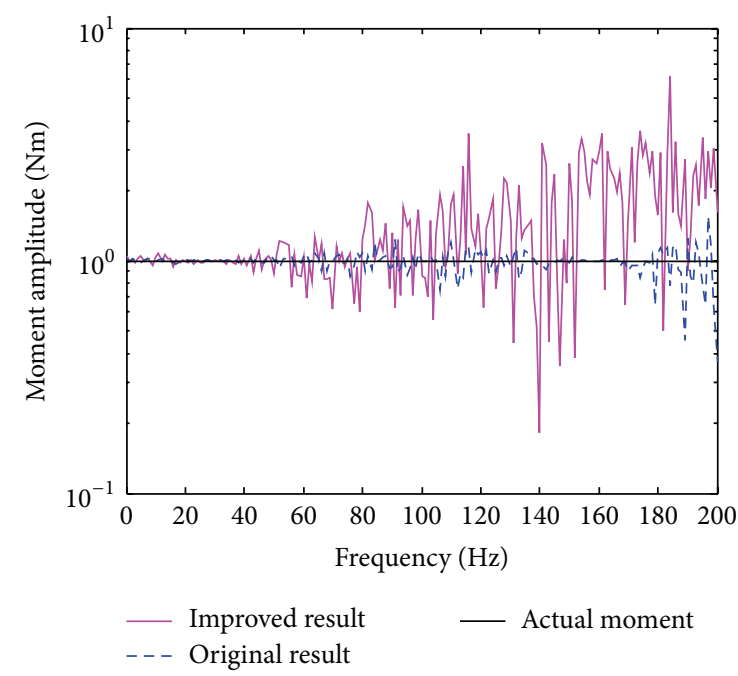

(f)

FIGURE 6: Comparison of improved results and original results. (a) Force in $x$-direction. (b) Force in $y$-direction. (c) Force in $z$-direction. (d) Moment around $x$-direction. (e) Moment around $y$-direction. (f) Moment around $z$-direction. 


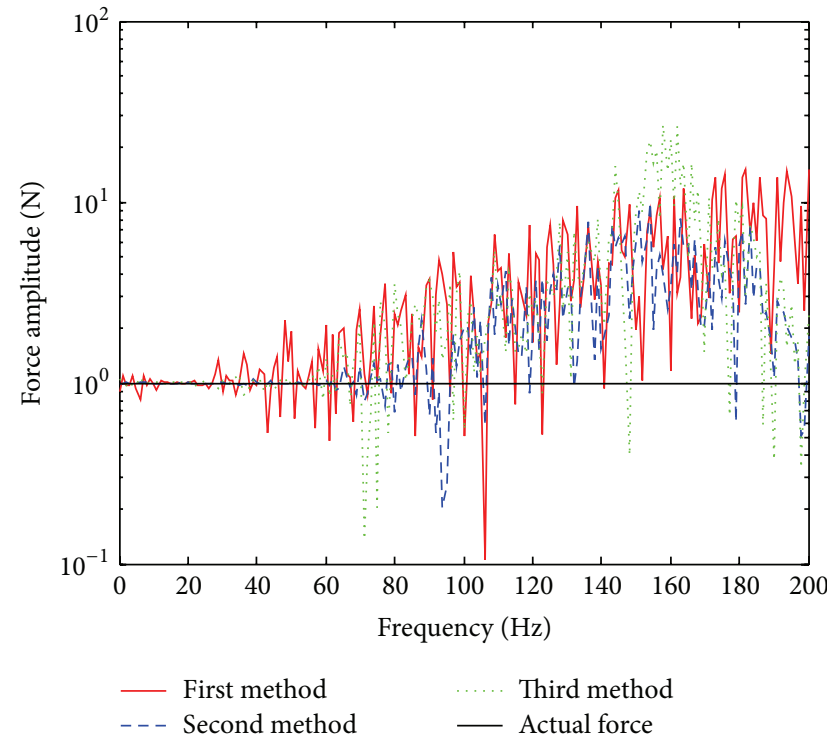

(a)

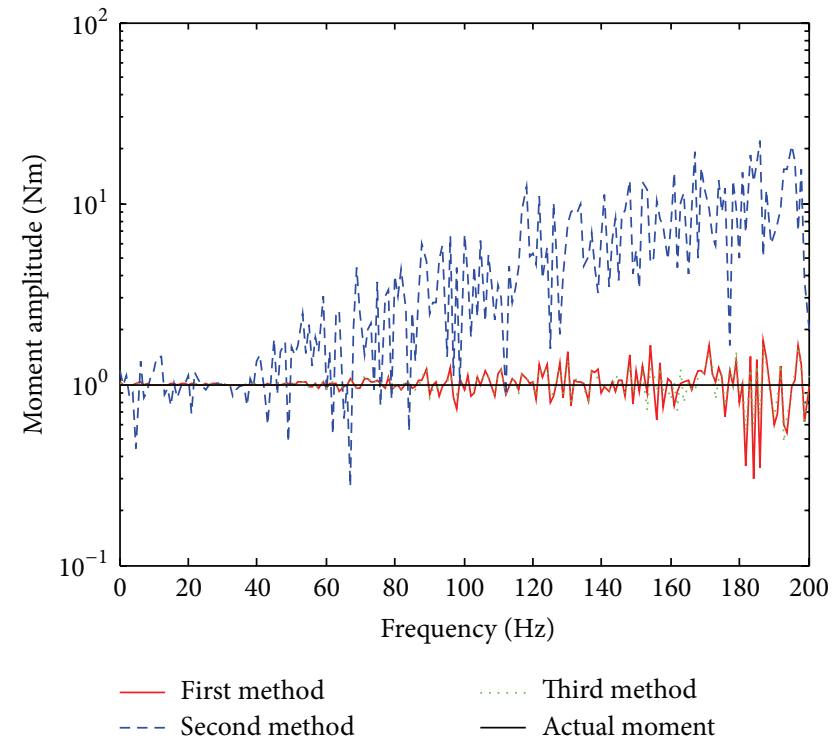

(b)

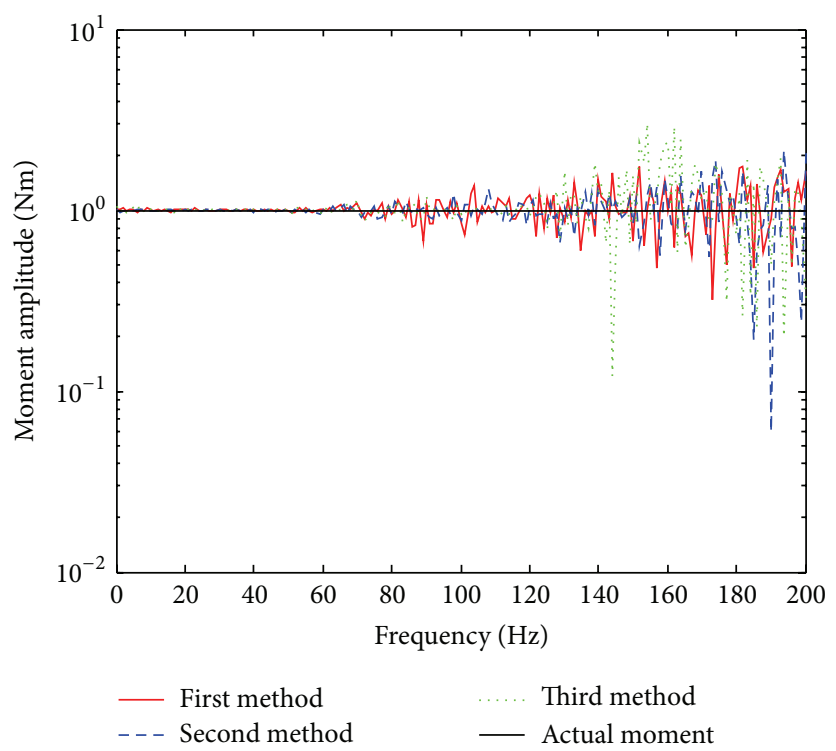

(c)

Figure 7: Comparison of estimated inputs by three different methods and actual inputs. (a) Force in $z$-direction. (b) Moment around $x$ direction. (c) Moment around $y$-direction.

excitation, the application of auto- and cross-power spectral density functions can effectively decrease measurement noise to improve reconstruction accuracy. Flexible thin plate is introduced as supporting structure in this work. However, the reconstruction method is suitable for not only this vibration isolation system but also other systems with variable supporting structures.

\section{Competing Interests}

The authors declare that they have no competing interests.

\section{Acknowledgments}

The first author would like to appreciate the financial assistance from China Scholarship Council.

\section{References}

[1] P. M. Frank, "Fault diagnosis in dynamic systems using analytical and knowledge-based redundancy: a survey and some new results," Automatica, vol. 26, no. 3, pp. 459-474, 1990.

[2] J. Wang and $\mathrm{H}$. Hu, "Vibration-based fault diagnosis of pump using fuzzy technique," Measurement, vol. 39, no. 2, pp. 176-185, 2006.

[3] N. R. Sakthivel, V. Sugumaran, and S. Babudevasenapati, "Vibration based fault diagnosis of monoblock centrifugal pump using decision tree," Expert Systems with Applications, vol. 37, no. 6, pp. 4040-4049, 2010.

[4] J. C. Snowdon, "Vibration isolation: use and characterization," The Journal of the Acoustical Society of America, vol. 66, no. 5, pp. 1245-1274, 1979. 
[5] F. D. Bartlett Jr. and W. G. Flannelly, "Model verification of force determination for measuring vibratory loads," Journal of the American Helicopter Society, vol. 24, no. 2, pp. 10-18, 1979.

[6] S. E. S. Karlsson, "Identification of external structural loads from measured harmonic responses," Journal of Sound and Vibration, vol. 196, no. 1, pp. 59-74, 1996.

[7] S. Granger and L. Perotin, "An inverse method for the identification of a distributed random excitation acting on a vibrating structure part 1: theory," Mechanical Systems \& Signal Processing, vol. 13, no. 1, pp. 53-65, 1999.

[8] C. W. Groetsch, Inverse Problems in the Mathematical Sciences, Springer, 1993.

[9] P. A. Nelson and S.-H. Yoon, "Estimation of acoustic source strength by inverse methods: part I, conditioning of the inverse problem," Journal of Sound and Vibration, vol. 233, no. 4, pp. 639-664, 2000.

[10] E. Jacquelin, A. Bennani, and P. Hamelin, "Force reconstruction: analysis and regularization of a deconvolution problem," Journal of Sound and Vibration, vol. 265, no. 1, pp. 81-107, 2003.

[11] P. Schevenels, P. J. G. Van Der Linden, and G. Vermeir, "An inverse force measurement method to determine the injected structure-borne sound power from an installation into a building element," Building Acoustics, vol. 17, no. 3, pp. 199-219, 2010.

[12] T. Uhl, "The inverse identification problem and its technical application," Archive of Applied Mechanics, vol. 77, no. 5, pp. 325337, 2007.

[13] M. H. A. Janssens, J. W. Verheij, and T. Loyau, "Experimental example of the pseudo-forces method used in characterisation of a structure-borne sound source," Applied Acoustics, vol. 63, no. 1, pp. 9-34, 2002.

[14] H. Inoue, N. Ikeda, K. Kishimoto, T. Shibuya, and T. Koizumi, "Inverse analysis of the magnitude and direction of impact force," JSME International Journal, Series A: Mechanics \& Material Engineering, vol. 38, no. 1, pp. 84-91, 1995.

[15] T. P. Nordberg and I. Gustafsson, "Using QR factorization and SVD to solve input estimation problems in structural dynamics," Computer Methods in Applied Mechanics and Engineering, vol. 195, no. 44-47, pp. 5891-5908, 2006.

[16] A. N. Tikhonov, "Solution of incorrectly formulated problems and the regularization method," Soviet Physics Doklady, vol. 4, pp. 1035-1038, 1963.

[17] T. S. Jang, H. Baek, S. L. Hana, and T. Kinoshita, "Indirect measurement of the impulsive load to a nonlinear system from dynamic responses: inverse problem formulation," Mechanical Systems and Signal Processing, vol. 24, no. 6, pp. 1665-1681, 2010.

[18] P. C. Hansen, "Regularization tools: a Matlab package for analysis and solution of discrete ill-posed problems," Numerical Algorithms, vol. 6, no. 1-2, pp. 1-35, 1994.

[19] C. Chang and C. T. Sun, "Determining transverse impact force on a composite laminate by signal deconvolution," Experimental Mechanics, vol. 29, no. 4, pp. 414-419, 1989.

[20] C.-H. Huang, "A nonlinear inverse problem in estimating simultaneously the external forces for a vibration system with displacement-dependent parameters," Journal of the Franklin Institute, vol. 342, no. 7, pp. 793-813, 2005.

[21] C.-H. Huang, "A generalized inverse force vibration problem for simultaneously estimating the time-dependent external forces," Applied Mathematical Modelling, vol. 29, no. 11, pp. 1022-1039, 2005.

[22] C. C. Paige and M. A. Saunders, "LSQR: an algorithm for sparse linear equations and sparse least squares," ACM Transactions on Mathematical Software, vol. 8, no. 1, pp. 43-71, 1982.
[23] W. L. Li and P. Lavrich, "Prediction of power flows through machine vibration isolators," Journal of Sound and Vibration, vol. 224, no. 4, pp. 757-774, 1999.

[24] J. Pan, J. Pan, and C. H. Hansen, "Total power flow from a vibrating rigid body to a thin panel through multiple elastic mounts," The Journal of the Acoustical Society of America, vol. 92, no. 2, pp. 895-907, 1992. 


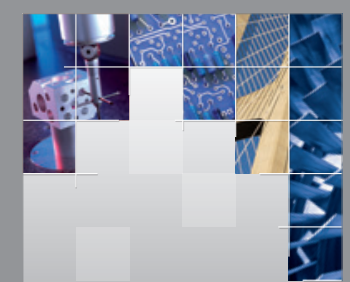

\section{Enfincering}
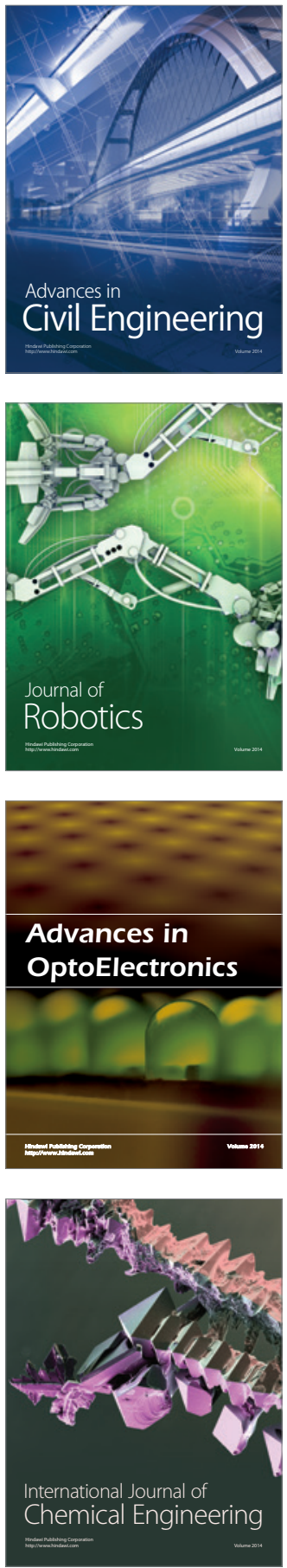

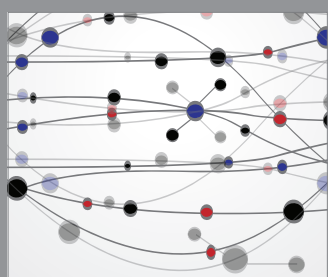

The Scientific World Journal

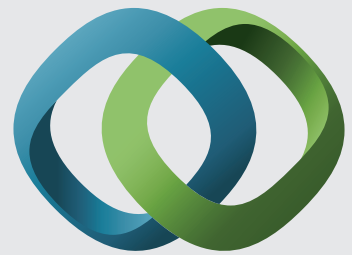

\section{Hindawi}

Submit your manuscripts at

http://www.hindawi.com
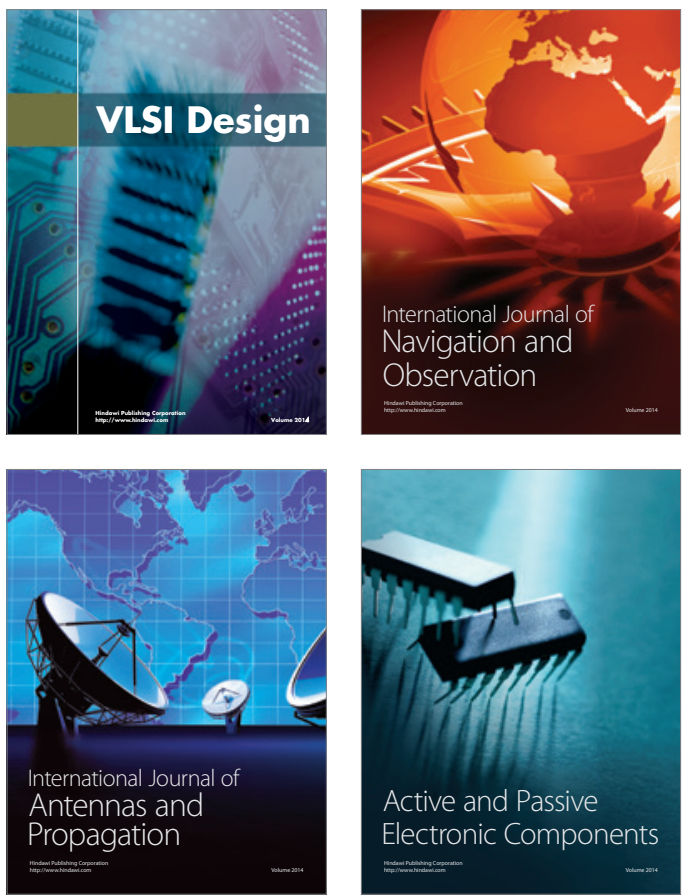
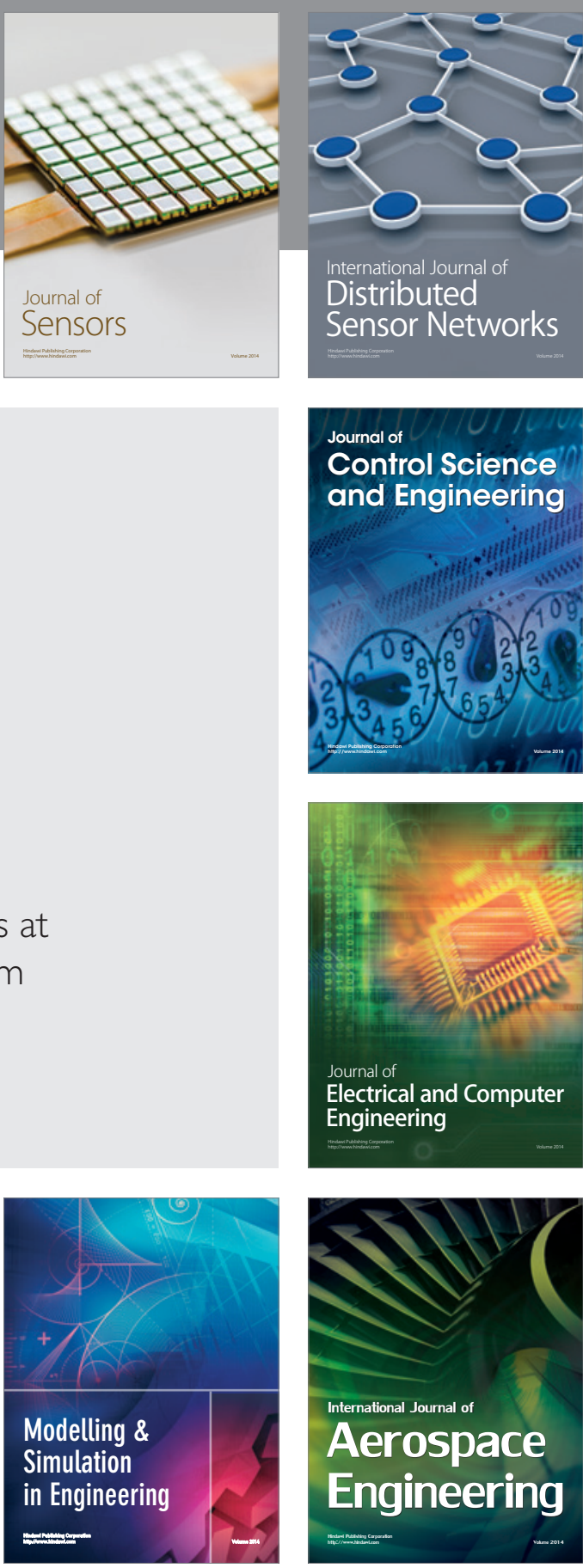

International Journal of

Distributed

Sensor Networks

Journal of

Control Science

and Engineering
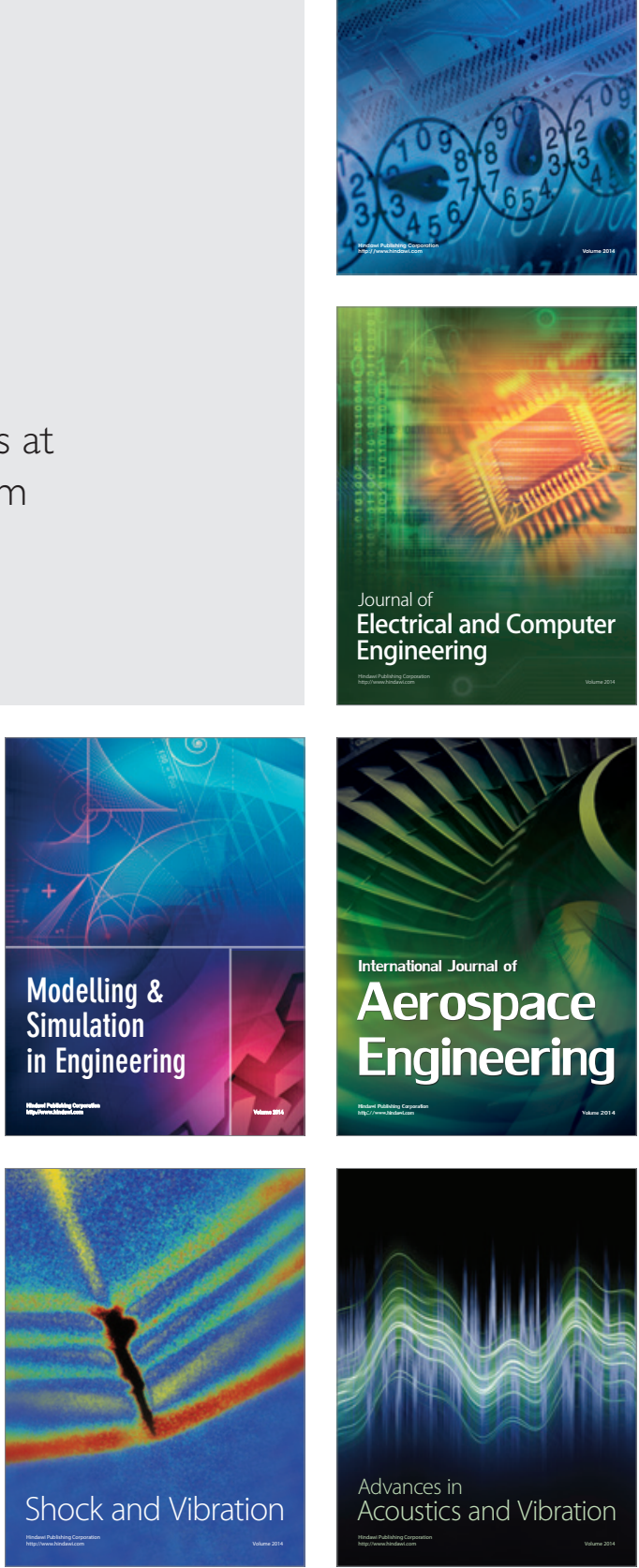